\title{
CAE Analysis Technology for Development Design Utilizing Statistical Sciences
}

\author{
M. Yamaji and K. Amasaka*
}

Aoyama Gakuin University, Japan

\begin{abstract}
In this paper, consideration is given to the validity of "statistical science", which is necessary for innovation of the development designing process, for use in a new issue involving numerical simulation technology (CAE) in development designing. This new application of statistical science is designed to aid the transition from the "prototype / experimental based real machine evaluation method" to the "prediction evaluation method based on highly reliable CAE analysis." More specifically, concrete examples of "statistical science study cases that contributed to CAE analysis" from automotive manufacturers are examined from the standpoint of "statistical science contributing to design science". This was done so as to propose the "highly reliable CAE analysis system approach method" that contributes to the process innovation of development designing.
\end{abstract}

\section{INTRODUCTION}

An urgent improvement of intelligent productivity in the advanced manufacturing processes of planning and development, designing, prototyping evaluation, mass production preparation, and mass production is vitally needed. This improvement is being done for the purpose of offering highly reliable products to create customer value in a short period of time [1]. Among other things, a close look at the development designing and production process stages reveals excessive repetition of "experiment, prototyping, and evaluation". This repetition prevents the "scale-up effect" generated in the bridging stage between prototyping, experiment, evaluation, and mass production. Therefore, innovation of the development and production method, as well as reduction of the development period, is a top priority issue. The automotive industry is now attempting to realize a "global production strategy for simultaneous achievement of QCD (quality, cost and delivery)" that aims to achieve "worldwide uniform quality and production at optimum locations", in an effort to prevail and survive in the "worldwide quality competition" [2].

What is urgently needed is innovation that will promote the advancement from the conventional evaluation-based development, which uses the prototyping and experiment process (a method based on the confirmation of real goods to attain improvement) that has long supported highly reliable designing, to a CAE (Computer Aided Engineering) prediction-based designing process for strategic automotive development design.

Given this background, this paper demonstrates how "statistical science study cases that contributed to CAE analysis" were employed by automotive manufacturers in an effort to solve pressing technical problems. Then, through verification of these examples, it proposes the "highly reliable CAE analysis system approach method" that will con-

"Address correspondence to this author at the School of Science and Engineering, Aoyama Gakuin University, 5-10-1, Fuchinobe, Sagamihara-shi, Kanagawa-ken, 229-8558 Japan; Tel: +81-42-759-6313; Fax: +81-42-7596556; E-mail: kakuro_amasaka@ise.aoyama.ac.jp tribute to the innovation of the process of development designing [1-3].

\section{CAE ANALYSIS TECHNOLOGY FOR DEVELOP- MENT DESIGN UTILIZING STATISTICAL SCIENCES}

In this study, the establishment of the "high quality assurance CAE model", which is expected to simultaneously realize QCD, is being promoted. This is being done with the validity of "statistical science [4]" as the basis and in response to the super short period of automotive development and designing. To be more specific, from the standpoint of Verification / Validation (divergence of CAE from theory / divergence of CAE from experiments), and for the purpose of attaining a highly reliable CAE analysis that is consistent with the market - experiment - theory profile, first, (i) a "high quality assurance business process model - the Total QA High Cycle-ization Process Model," that contributes to quality improvement in the development designing process, was developed [4,5]. Next, a highly reliable CAE analysis model, the "Total Intelligent CAE Management Model", which is designed to interpret the failure mechanism as indicated in Fig. (1) $[1,4]$ was also developed. Through the application of these dual models, the establishment of a prediction evaluation that is based on the high quality assurance CAE model will be made possible.

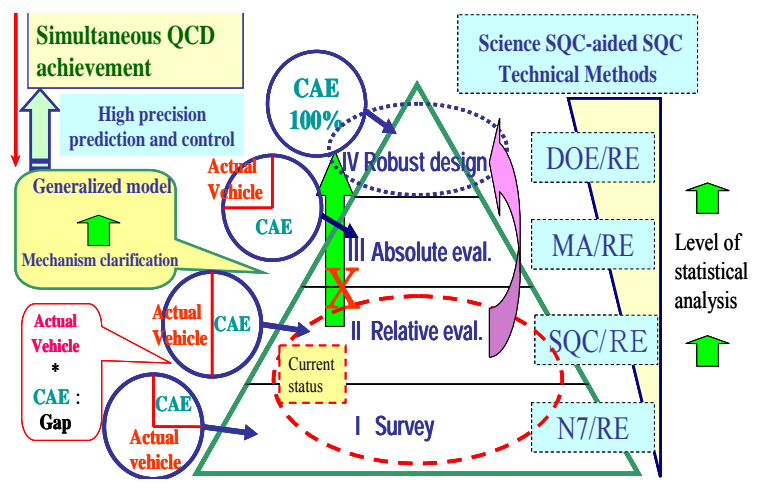

Fig. (1). Total Intelligent CAE Management Model. 


\subsection{Statistical Science Contributing to Design Science}

In recent years, the utilization of simulation technology that aims to accelerate the field of product development and design has been gathering attention. It is necessary to give consideration to the evolutionary applications of design from the experiment method. In other words, effective implementation of numerical experiments and the concept of robust design are becoming more necessary. Therefore, error factors, a fundamental method used even for statistical science based simulation, were introduced. This will involve the incorporation of statistical science into the latest simulation technology (CAE) at the development and designing stage. As a result, (1) the possibility of "realizing cost reduction during high quality development and shortening of the production development period" will be demonstrated, and (2) the validity of statistical science will be improved through transverse and systematic study activities. This will be done for the purpose of eliminating the concern that "development and designing by CAE tends to rely on the different personal characteristics of those who conduct it."

\subsection{Statistical Science Contributing to Problem Solving in the Development Design Stage}

Generally speaking, the uses of statistical science that are expected to solve technical problems are (i) the search for alternative characteristics, (ii) the extraction of factors, (iii) the clarification of mechanisms, (iv) the structuring of prediction models, and (v) the verification of optimization, and so on. For this reason, basic statistical science, experiment planning, multivariable analysis, reliability analysis, the optimization method, and time series analysis, among others, are selected according to necessity, and then combined and utilized [6].

The main focus of statistical science being utilized by the development and design staff is shown through the following three cases.

(i) The first is variation factor analysis. This is a case where many of the design factors that constitute the structure of the problem are elucidated as "empirical knowledge" and identified in a technological manner in order to solve the problem. However, there are cases where the contribution rate of these solutions can not be made abundantly clear. Statistical science will contribute to search for design specification factors and optimal standards.

(ii) The second is identification and control of the principal design factors to solve the problem quickly. In this case, the structure of technological problems can be technically surmised, but they exist in a technological domain which is crowded with design factors. Therefore, it is expected that the unknown contribution rate of the design factors should be investigated further, the countermeasure factors should be identified, and the results controlled by means of variation factor analysis utilizing statistical science. For statistical science analyses conducted in recent years, curve fitting (or polynomial approximation technique) is utilized as an approximate means of accomplishing this.

(iii) Third is modeling that can be carried out for structural identification and prediction of the cases where the structural mechanism of the technological problems is unknown. This is most useful for finding the "solution to new technological problems" for which empirical knowledge or proper technology has not been sufficiently developed, or the "solution to bottle-neck technological problems" which are still pending. In this case it is necessary to take a demonstrative scientific approach to identify the dynamic behavior at the time that the problem occurs through the use of visualization techniques. This will clarify the structure (mechanism) of the problem, and then allow for rigorous modeling of the cause-and-effect relationship that is present. Statistical science will contribute to clarify the mechanism and modeling of this.

\section{HIGHLY RELIABLE CAE ANALYSIS SYSTEM APPROACH METHOD}

When performing CAE analysis (as shown in Fig. 1), in the case where the highly precise absolute value evaluation is expected to be consistent with the real machine and experiments, modeling for prediction and control is required for the rigid cause-and-effect relationship. A mathematical and universal structural model is required and proper high-level technology (clarification of the mechanisms where the problems occur) holds the keys to success [7, 8].

On the other hand, with regard to surveillance or relative evaluation, which complements the results from real machines or experiments, modeling for prediction and control in factor analysis is expected to probe the factors and standards for variation of objective variables, and a rigid cause-andeffect relationship is not necessary. Consequently, CAE analysis software is generally modified (or simplified) to generate approximate solutions considering such factors of convenience as usability, sustainability, and feasibility. Consequently, such software design policy is the mainstream today.

Given the above, the authors hereby propose the "Highly Reliable CAE Analysis System Approach Method" as indicated in Fig. (2). This methodology is designed to conduct CAE analysis through the use of three case methods (I, II, and III) to find the solutions to various problems related to development and designing.

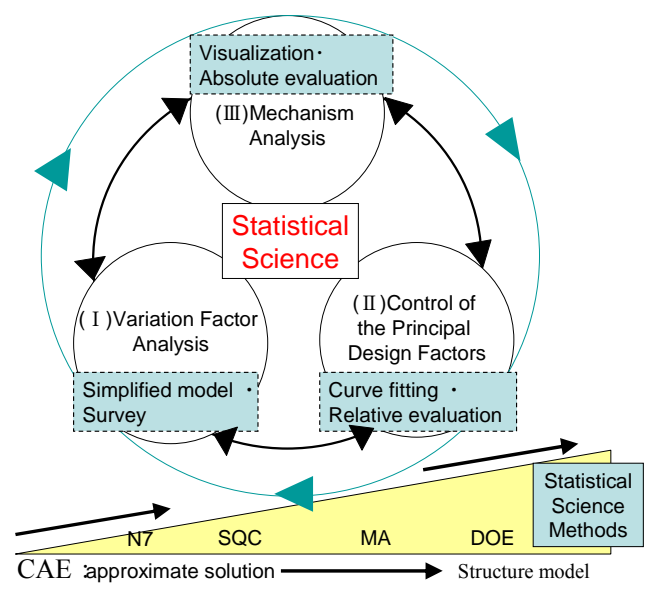

Fig. (2). Highly Reliable CAE Analysis System Approach Method.

(I) A case where the design factors are identifiable as unique technologies.

Case Method (I) is a CAE analysis system approach method which utilizes statistical science focusing on "varia- 
tion factor analysis" to make effective use of unique technologies. This case is generally implemented as a simplified model that contributes to the supplementary "survey" used for the prototyping and experimenting processes indicated in Fig. (1).

(II) A case where the design factors are technologically unknown.

Case Method (II) is a "relation between CAE statistical science" approach in the case where the mechanism of the problem that occurs can be surmised to a certain extent through the use of unique technology. However, it is not sufficiently clear, from a technological standpoint, how the design factors are contributing to the results obtained. This is partly because of the complicated mutual interactions between a large variety of factors and a lack of empirical technology. In the cases of univariate actual machine experiments, such design factor effects are often not identified with high precision, so it is realistic to conduct curve fitting by means of a neural network ( $\mathrm{NN}$, or nonlinear multiple regression analysis method) so as to reduce the deviance between the actual machine results and those of the CAE analysis. This case is considered to be useful for a wide range of applications as a methodology contributing to the "relative evaluation" found in Fig. (1), rather than for the rationality or rigidity of the model equations obtained.

(III) A case where the mechanism of the technological problems is unknown.

Case Method (III) proposes using a "relation between CAE statistical science" approach in the case where the "mechanism of technological problems", the issue the development designers are most interested in, is not very well understood. This is done with a view to reflecting it specifically in actual business processes through the means of research and verification.

Here, the "Highly Reliable CAE Analysis System Approach Method", is used not only to "grasp the problem phenomena", but to get an accurate grasp of the "real cause of the problem that occurs and its mechanism". This method constitutes the basis for solving relevant technological issues.

As a result, a highly reliable CAE system approach, the "Total Intelligence CAE Management System Approach" as shown in Fig. (3) was created. This approach realizes the "creation of high precision CAE analysis software" and accomplishes the simultaneous achievement of QCD [9-11].

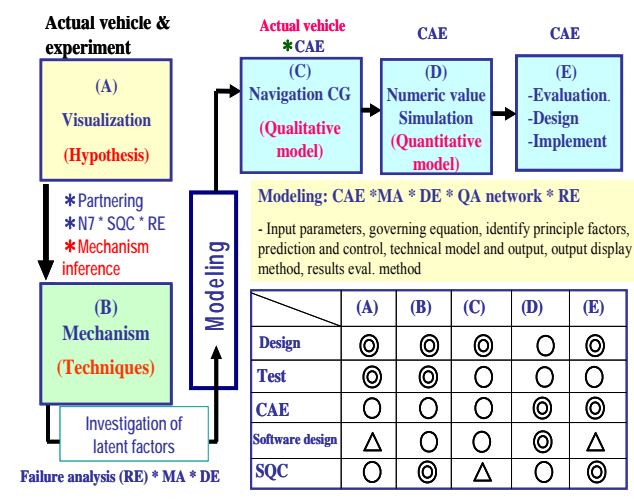

Fig. (3). Total Intelligence CAE Management System Approach.
As seen in the figure, first, (A) the actual vehicle and experiment (meaning bench evaluation tests using actual vehicles and parts) visualizes the dynamic behavior (tricky mechanism) of the problems. Next, by means of (B) the factorial analysis in which the unique technology and empirical technology are combined together with N7 (New 7 QC Tools), SQC (Statistical Quality Control), MA (Multivariate Analysis), and DOE (Design of Experiments), the latent factors which cause the problem are investigated using actual vehicles and experiment procedures in an effort to clarify the problem mechanism.

Based on the knowledge obtained through the above steps, as well as the navigation process using CG (Computer Graphics) created through a combination of (C) experiments and $\mathrm{CAE}$, qualitative modeling of the problems was conducted. Furthermore, for the purpose of accurately reproducing the problem mechanism, which has been grasped through an inductive approach using visualization experiments, quantitative modeling is conducted. This is done by means of (D) numeric value simulation. In the final stage, the (E) differential (gap) between the evaluation results of actual vehicles and experiment procedures (absolute value) and those of the CAE analysis (simulation value) was confirmed.

The target differential ratio for the analysis precision is between $1 \%$ and $2 \%$. To achieve such a target, an all-out, collective partnering between the chief engineers (double circle), collaborating engineers (circle), and assistants (triangle) who are involved in the design, testing, CAE analysis, CAE software development, and SQC throughout the process stages from (A) to (E) indicated in the figure is essential.

In the next chapter, a few examples are discussed where the development and design staff employ statistical science in an effort to conduct effective CAE analysis. By considering the ways that such statistical science can be utilized, the "highly reliable CAE analysis system approach method", which can contribute to the process innovation of product development and design is proposed.

\section{APPLICATION EXAMPLES}

\subsection{Cases Where Design Factors are Identifiable Through Proper Technology: Examples of Applying the "SQC Technical Method" to Optimal CAE Anti- Vibration Design for Door Outer Mirrors}

First, an anti-vibration design approach method using $\mathrm{CAE}$ analysis is discussed here for the prevention of "mirror vibration" by the outer mirrors of trucks [9]. The prevention of "mirror vibration" is important to ensure the field of view to the front and back of the truck, as well as to the right and left. This requires "optimization of the anti-vibration structure (simultaneous achievement of QCD)" by means of applying $\mathrm{CAE}$ at a minimum cost. For the case of conducting anti-vibration design without using trial and error, the CAE analytic approach method statistical science Technical Method [4,6] is applied as shown in Fig. (4). This was done via a cause analysis approach method for the "mirror vibration" based on the accumulated proper technology.

In order to conduct CAE analysis without divergence from the real machine and experiment results (to search for design specification factors and optimal standards that will 


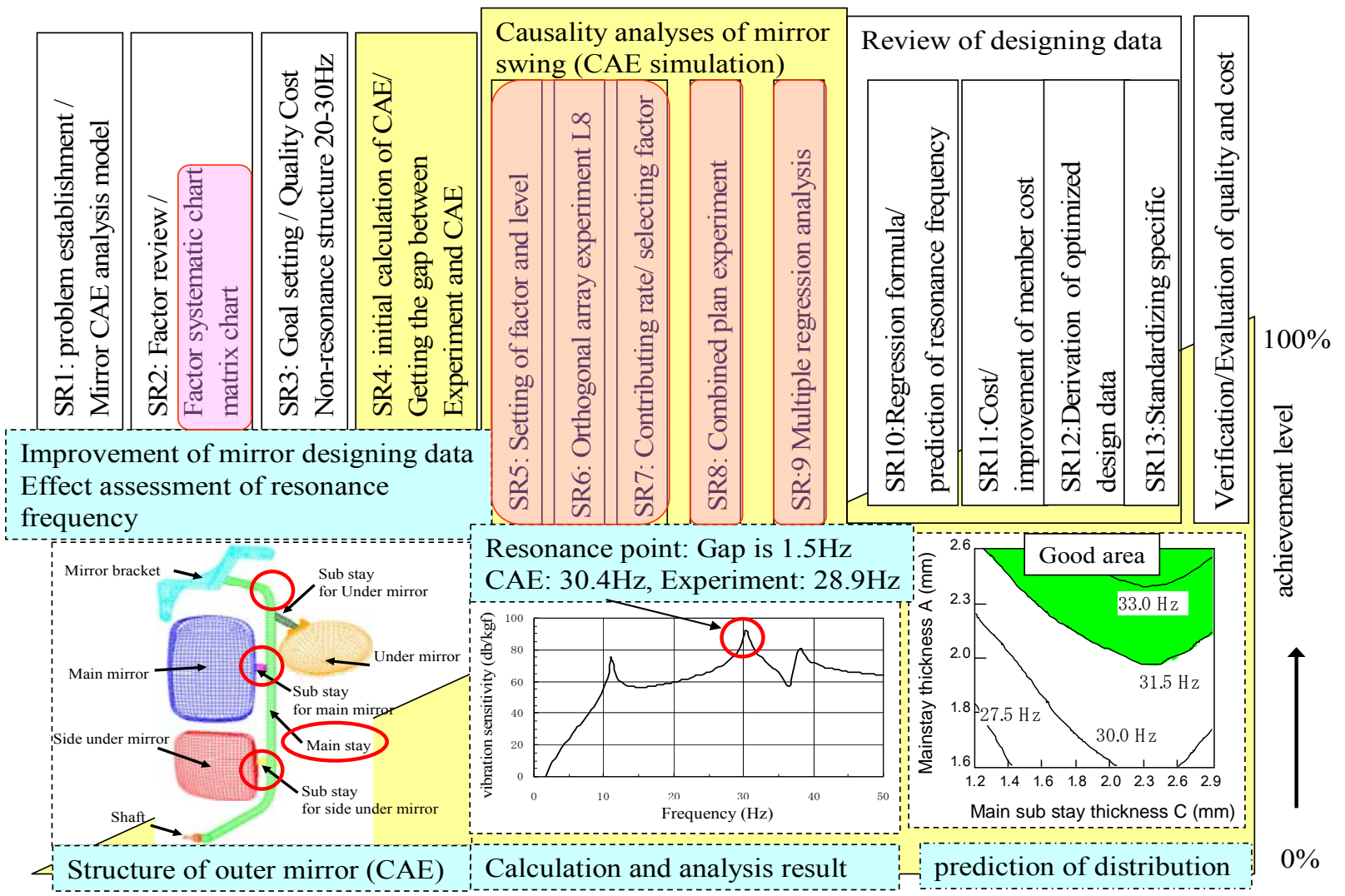

Fig. (4). The CAE analytic approach method SQC Technical Method.

contribute strongly to anti-vibration measures), a simulation will be carried out that utilizes statistical science. Then the resonant frequency which prevents mirror vibration will be predicted and controlled in an effort to improve the design specifications (for weight and structure).

As shown in Fig. (4), (SR1) issue setting, (SR2) factor consideration (N7: factor schematic, matrix diagram, etc.), and (SR3) target setting (non-resonant point over $30 \mathrm{~Hz}$ ) will be conducted as steps in the process of improving the antivibration performance. At the stage of CAE initial calculation (SR4), a difference (gap) between the resonant frequency obtained from the experiment results and the frequency obtained from the CAE values will be ascertained from the deformation taking place mainly at three points in the mainstay holding the main mirrors (which are vibrating up and down). In the cause analysis stage (CAE simulation) (SR5) the setting of factors and standards (plate thickness, the diameter of the mainstay and side sub-stays, five factors and interactions) will be conducted. An orthogonal array experiment (L8: two standards system) will be conducted (SR6) and then (SR7) the factors contributing most to the problem will be selected. Moreover, taking into consideration the secondary effects of the selected factors, (SR8) a composite designed experiment will also be carried out. Using the experiment data, (SR9) multiple regression analysis is conducted for the purpose of curve fitting in consideration of the primary and secondary effects. Next, by applying (SR10) the derived regression formula, consideration is given to the design specifications. In (SR11) the aim is to balance the performance with the cost, and in (SR12) the resonant frequency contour distribution prediction will be conducted to derive the optimal design specifications. In the figure is an example of mainstay thickness and sub-stay thickness. By using the approach described above, scrapand-build modifications can be implemented for multiple specification stays, while enabling (SR13) a balance between the performance and cost to be maintained. This will accelerate the process to move to the production stage and simultaneous QCD will be accomplished.

\subsection{Cases Where Design Specification Factors are not Clarified: Estimation of Lift Characteristics Through Parallel use of a Neural Network (NN) and Multiple Re- gression Analysis}

In the development and design stage, there are many cases where cause-and-effect relations are too complex to be solved only with the accumulated technology. One such case in which the design specification factors needed for cause analysis are not clearly identified is "modeling of prediction and control of lift characteristics" using a real vehicle sub-

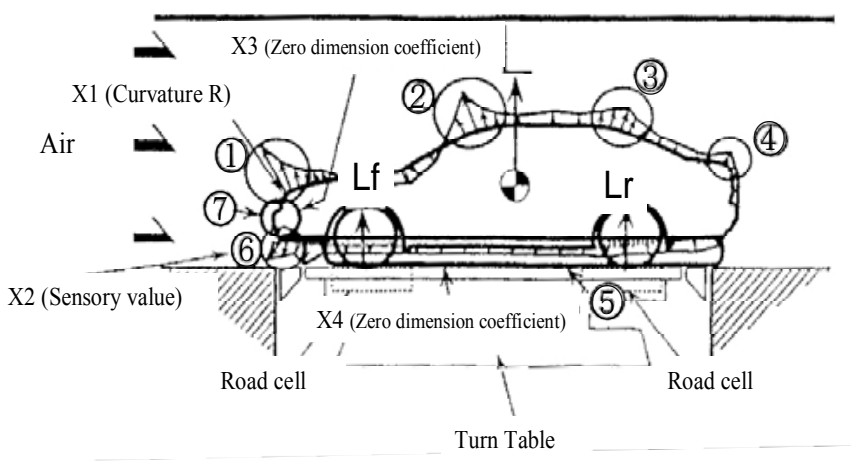

Fig. (5). Conditions in a wind tunnel and the pressure distribution of the vehicle's center. 


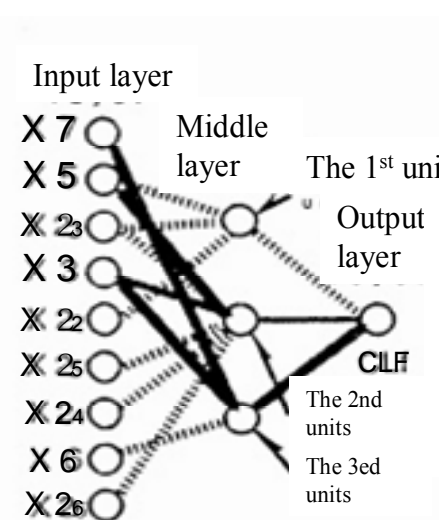

Solid line: positive correlation Broken line: negative correlation

(a) Neural network structure

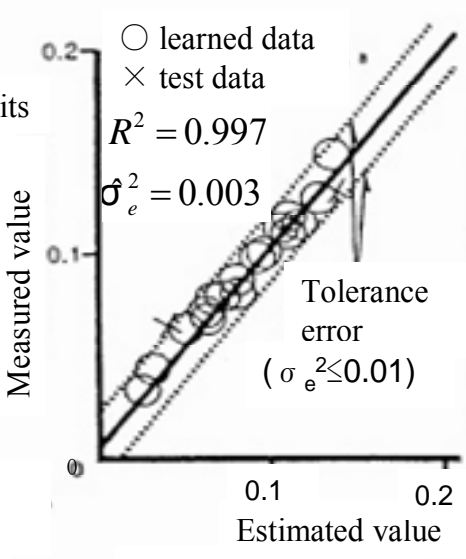

(b) Re-analysis result

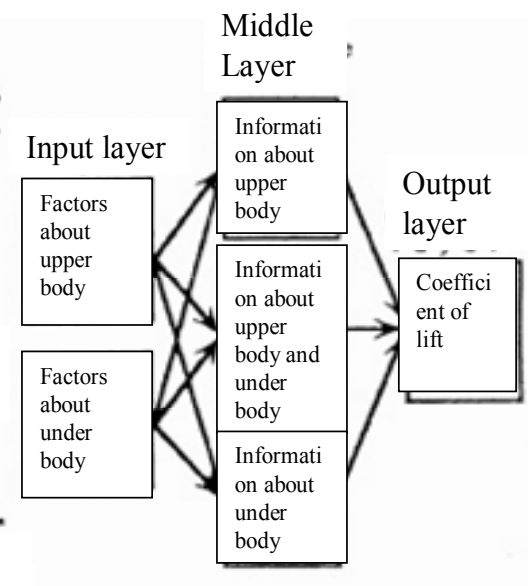

(c) Relation between each NN's layer

Fig. (6). Analysis via a neural network.

mitted to wind tunnel testing [12]. Fig. (5) illustrates a large wind tunnel used for measuring the lift of an actual vehicle. Load cells are embedded at the tire grounding points in the wind tunnel to enable the longitudinal, vertical, and transverse forces to be measured. The lift is expressed as the vertical force applied to the load cells, and is generally measured at a wind velocity equivalent to $120 \mathrm{~km} / \mathrm{h}$.

In this case, through use of multiple regression analysis (sequential analysis, partial regression plot, and so on), in addition to the upper body shape factor of the vehicle, the lower body shape factor was also found to be another new relevant design factor, and the interactions between these composite factors were also identified. Through close examination of the multiple regression model that was produced, the mechanism of the lift characteristics was estimated and confirmation was obtained through real machine experiments.

Using the knowledge gained from this analysis, curve fitting was efficiently conducted by employing a NN [13] of nonlinear regression analysis.

Fig. (6) (a) illustrates the neural network calculated after re-learning based on all explanatory variables and the designing factors obtained by multi-regression analysis. Fig. 6(b) illustrates the relationship between the lift coefficient and the NN calculated value. Fig. 6(c) illustrates the causal relationship between the lift coefficient and the designing factors.

This study on testing the methods and results so far obtained contribute to shortening the research and development period.

\subsection{Cases Where the Mechanism of technical problems is unclear: High Precision CAE Analysis of Oil Leakage at the Drive-Train Oil Seal}

In this study, a devise was developed for visualizing the "oil leak" from the oil seal [14-16] on the surface of a high rotation drive shaft for the purpose of clarifying its mechanism (cause and effect) $[1,5,17,18]$. Through incorporation of the "SQC Technical Method"[19], a cause analysis was conducted through real machine experiments by means of parallel use of reliability engineering and time series analysis. In this project, an organizationally transverse team was formed gathering intellectuals from both inside and outside the company, where the development and design department staff worked together with CAE manufacturers, SQC experts, specialists on real machine experiments and $\mathrm{CAE}$ analysis, and so on.

\subsubsection{The New Approach Utilizing Statistical Science}

To clarify the oil seal leakage mechanism for transaxles, Statistical Science [19] was implemented with a mountainclimbing-type problem solution technique and using the "SQC technical methods" [20], as indicated in Fig. (7). The three elements of "Management SQC" [21], "Technology Management [TM]", "Production Management [PM]", and "Information Management [IM]" were developed as indicated in each stage of the team activity process.

As the figure illustrates, both teams are linked through the implementation of "Management SQC" [20] by combining the three management methods. In addition, the mountain climbing method for problem solving utilizing the "SQC Technical Methods" [20] was used to achieve improvement in terms of both design reliability and production quality.

Accordingly, a device was developed to visualize the dynamic behavior of the oil seal lip in order to turn this "unknown mechanism" into explicit knowledge, as shown in Fig. (8). As shown in the figure, the oil seal was immersed in the lubrication oil in the same manner as the transaxle, and the drive shaft was changed to a glass shaft that rotated eccentrically via a spindle motor so as to reproduce the operation that occurs in an actual vehicle. The sealing effect of the oil seal lip was visualized using an optical fiber.

It was conjectured that in an eccentric seal with one-sided wear, foreign matter becomes entangled at the place where the contact width changes from small to large. Two trial tests were carried out to ascertain if this was true or not. Based on the observation of the returned parts from the market and the results of the visualization experiment, it was observed that very fine foreign matter (which was previously thought as not impacting the oil leakage) grew at the contact section, as shown in (Fig. 9A) (Test-1). 


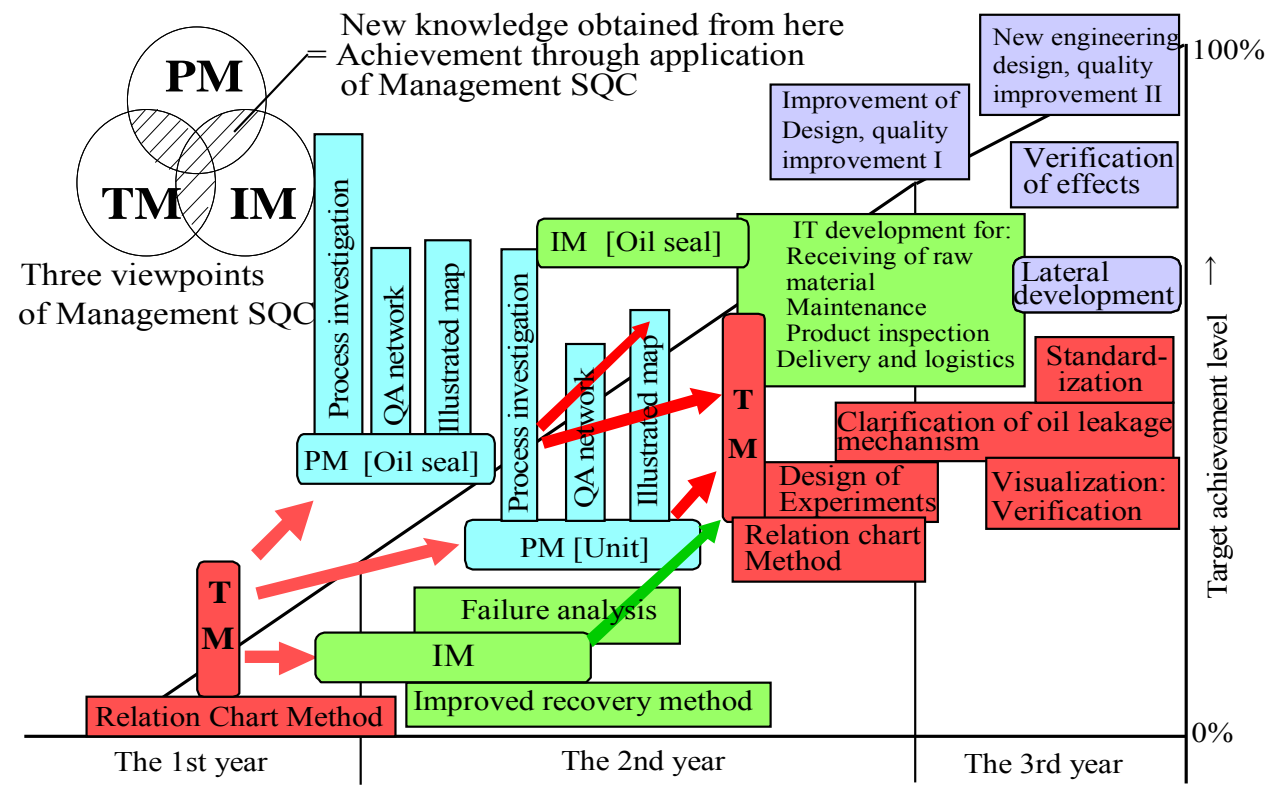

Fig. (7). The Mountain Climbing Type of Problem Solving Utilizing SQC Technical Methods.
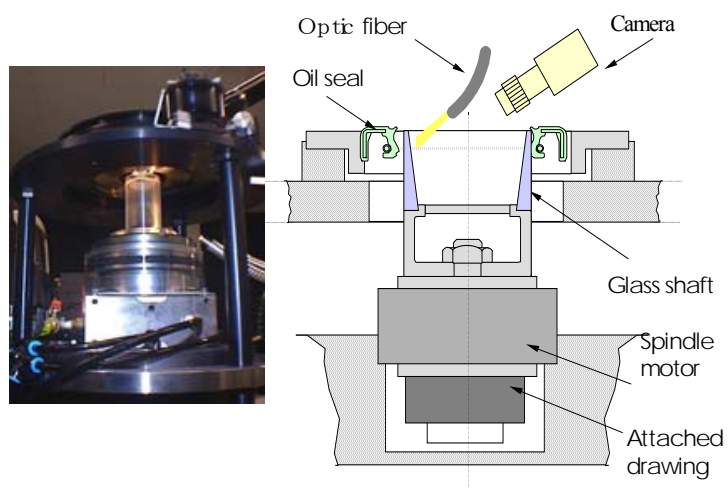

Fig. (8). Outline of Oil Seal Visualization Equipment.

From a result of the component analysis, it was confirmed that the fine foreign matter was the powder produced during gear engagement inside the transaxle gear box. This fine foreign matter on top of microscopic irregularities on the lip sliding surface resulted in microscopic pressure distribu- tion which eventually led to the degrading the sealing performance, as shown in Fig. (9B) (Test-2).

A survey was conducted along with an experiment as described below:

First, in addition to defective oil seals, non-defective ones were also collected on a regular basis to check if the oil leak could be reproduced and to make comparisons through visual observations. Next, transaxle units from vehicles, with and without oil leak problems, were also collected on a regular basis to check if the leak could be reproduced in the same way. Integrating the results from the transaxles with and without defective oil seals confirmed that the defect could be reproduced. In all of these tests, oil leaks were reproduced as expected. Based on these test results, a Weibull analysis was then conducted.

As a result of this analysis, the cumulative number of oil leak claims by production month decreased to one twentieth of the original level [18]. The final outcome of achieving longer oil seal life was achieved as initially planned.

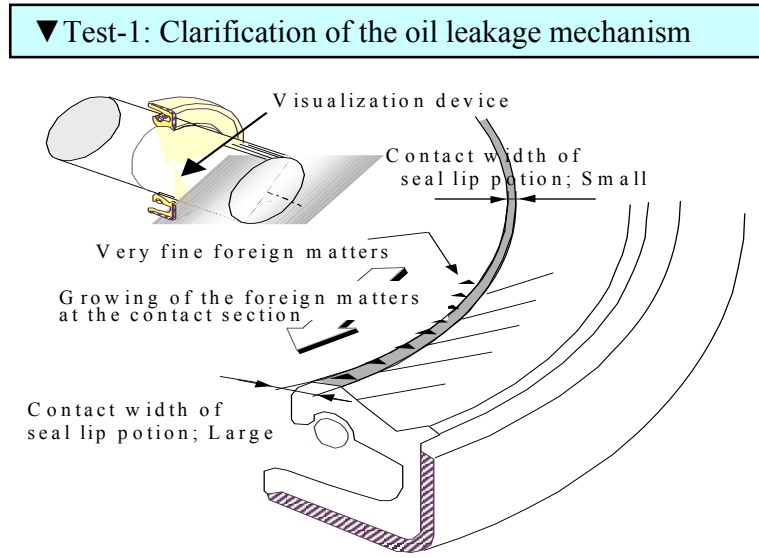

Fig. (9A). Test-1 Result of Oil Leakage Mechanism.
V Test-2: Even fine foreign matter can be accumulated to grow

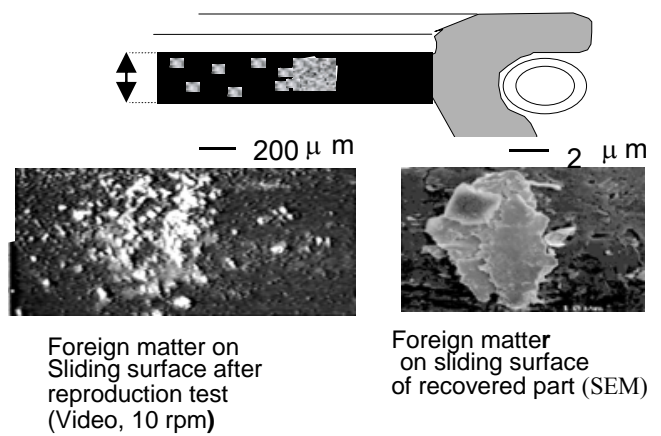

Fig. (9B). Test-2 Result of Oil Leakage Mechanism. 


\subsubsection{Intelligence CAE Software - Numerical Simulation by CAE to Solve the Oil Leakage Problem}

This clarified concept of conducting Numerical Simulation by CAE - The Sliding Surface Model has been applied to the development design engineering of high precision oil seals. Specifically, as a result of incorporating the Intelligence CAE Software, the minute roughness on the sliding surface has been controlled by regulating the composition of materials used. The next factor concerned the biased distribution of roughness toward the oil side of the seal. This can be interpreted as the bias of contact pressure distribution toward the oil side. Therefore, this factor has been controlled through the application of shape designing technology to design the seal lip more precisely.

The results obtained, from incorporating CG Navigation and Intelligence CAE Software-OL - Analysis (Fig. 10) to improve the high precision sealing mechanism of oil seals through the study conducted by the authors, have been established as a predictive engineering method for functional designing of oil seal parts. These results have also been applied to improvements in development design engineering for engine parts with oil seal leakage-related problems and with similar positive results were obtained.

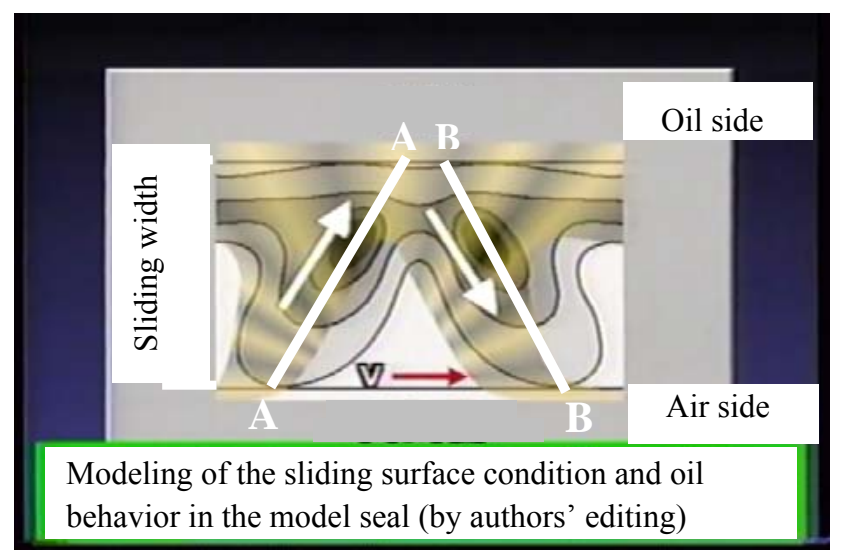

Fig. (10). CG Navigation and Intelligence CAE Software-OL.

\section{CONCLUSIONS}

In this paper, consideration was given to the validity of "statistical science", which is necessary for innovation of the development designing process, for use in a new issue involving numerical simulation technology (CAE) in development designing. More specifically, concrete examples of "SQC study cases that contributed to CAE analysis" from automotive manufacturers were examined. These cases were examined from the standpoint of "the transverse characteristics of statistical science and their contribution to design science" in order to propose the "highly reliable CAE analysis system approach method" that contributes to the innovation of the development designing process.

\section{REFERENCES}

[1] K. Amasaka and M. Yamaji, "Advanced TDS, Total development design management model", in The International Business \& Economics Research Conference, 2006, pp.1-15.

[2] K. Amasaka, "Highly reliable cae model, the key to strategic development of new JIT", Journal of Advanced Manufacturing Systems, vol. 6, no. 2, 159-176, 2007.

[3] M. Yamaji, T. Tanabe, H. Tsubaki and K. Amasaka, "Proposal and implementation of the high reliability CAE model", in International Conference on Reliability Maintainability and Safety, 2007, pp.1-7.

[4] K. Amasaka, Science SQC, New Quality Control Principle, Springer, 2004.

[5] K. Amasaka, "An intellectual development production hyper-cycle model in toyota", in the Production and Operations Management Society, Boston, 2004, pp.1- 20.

[6] K. Amasaka, "Proposal and implementation of the "Science SQC" quality control principle", International Journal of Mathematical and Computer Modeling, vol. 38 no.11-13, pp.1125-1136, 2003.

[7] J. De Vin Leo, H.C. Ng Amos and O. Jan, "Simulation-based decision support for manufacturing system life cycle management", Journal of Advanced Manufacturing Systems, vol. 3, no. 2, pp. 115$128,2004$.

[8] S. Strassburger, G. Schmidgall and S. Haasis, "Distributed manufacturing simulation as an enabling technology for the digital factory", Journal of Advanced Manufacturing Systems, vol. 2, no. 1, pp.111-126, 2003.

[9] H. Sue, S. Yamada, H. Tsubaki and K. Amasaka, "Interim report of WG4's studies in JSQC research activity of simulation and SQC: Effectiveness of statistical sciences for reformation of development design process (Part 1)", Journal of the Japanese Society for Quality Control, the 83rd Technical Conference, 2007, pp. 103-106, (in Japanese).

[10] T. Mitsuhashi, S. Takeoka, T. Tanabe, M. Yamaji and K. Amasaka, "Interim report of WG4's studies in JSQC research activity of simulation and SQC (The 4th Report): Concept of high reliability CAE for a fixed bolt (Part 2)", Journal of the Japanese Society for Quality Control, in the 83rd Technical Conference, 2007, pp. 107110 , (in Japanese).

[11] M. Yamaji, K. Amasaka, "High reliability CAE software model of developing the automotive development design, case study of high reliability CAE simulator for bolt tightening", in International conference of Risk, Quality and Reliability, 2007, pp. 2111-216.

[12] K. Amasaka, H. Nakaya, K. Oda, T. Oohashi and S. Osaki, "A study of estimating vehicle aerodynamics of lift -combining the usage of neural networks and multivariate analysis", The Institute of Systems, Control and Information Engineers, vol. 9, no. 5, pp. 229237, 1996.

[13] K. Funabashi, "On the approximate realization of continuous mappings by neural networks", Neural Networks, vol. 2, no. 3, pp.185$192,1989$.

[14] A. M. Lopez, K. Nakamura and K. Seki, "A study on the sealing characteristics of lip seals with helical ribs", in the 15th International Conference of British Hydromechanics Research Group Ltd. Fluid Sealing, 1997, pp. 1-11.

[15] Y. Sato, A. Toda, S. Ono and K. Nakamura, "A study of the sealing mechanism of radial lip seal with helical ribs -measurement of the lubricant fluid behavior under sealing contact", SAE Technical Paper Series, 1999.

[16] M. Kameike, S. Ono and K. Nakamura, "The helical seal: Sealing concept and rib design", Sealing Technology, no. 77, pp. 7-11, 2000 .

[17] K. Amasaka, Ed., New Japan Model Science TQM - Theory and practice of strategic quality management, Maruzen, 2007 (in Japanese).

[18] K. Amasaka, "A dual total task management team involving both toyota and NOK", in the Group Technology/ Cellular Manufacturing World Symposium, Columbus Ohio, 2003, pp. 265-270.

[19] K. Amasaka, "A demonstrative study of a new SQC concept and procedure in the manufacturing industry-establishment of a new technical method for conducting scientific SQC", The Journal of Mathematical \& Computer Modeling, vol. 3, no. 1, pp.1-10, 2000.

[20] K. Amasaka, "A study on "science SQC" by Utilizing "Management SQC"- A demonstrative study on a new SQC concept and procedure in the manufacturing industry" Journal of Production Economics, vol. 60-61, pp. 591-598, 1997.

[21] K. Amasaka, "Application of classification and related methods to SQC renaissance in toyota motor", Data Science, Classification, and Related Methods, Springer, 1998, pp. 684-695. 